
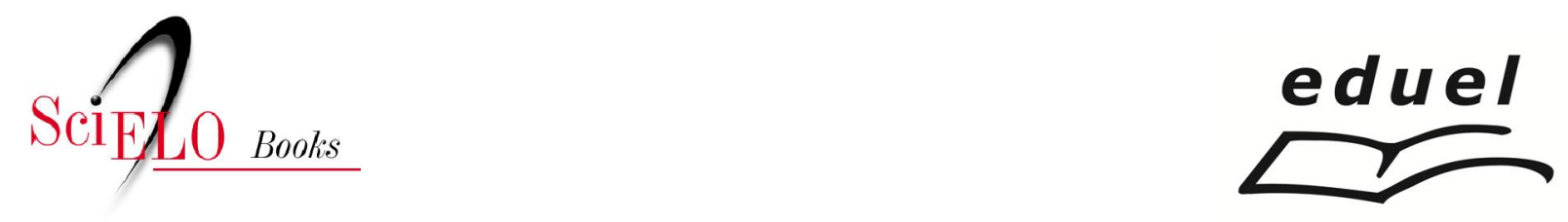

\title{
Prevenção de recaídas em dependência do tabaco
}

\author{
Sandra Odebrecht Vargas Nunes \\ Márcia Regina Pizzo de Castro \\ Carlos Roberto de Souza
}

\section{SciELO Books / SciELO Livros / SciELO Libros}

NUNES, SOV., CASTRO, MRP., and SOUZA, CR. Prevenção de recaídas em dependência do tabaco. In NUNES, SOV., and CASTRO, MRP., orgs. Tabagismo: Abordagem, prevenção e tratamento [online]. Londrina: EDUEL, 2011. pp. 119-130. ISBN 978-85-7216-675-1. Available from

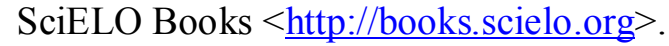

\section{(1)(0)}

All the contents of this work, except where otherwise noted, is licensed under a Creative Commons Attribution-Non Commercial-ShareAlike 3.0 Unported.

Todo o conteúdo deste trabalho, exceto quando houver ressalva, é publicado sob a licença Creative Commons Atribuição Uso Não Comercial - Partilha nos Mesmos Termos 3.0 Não adaptada.

Todo el contenido de esta obra, excepto donde se indique lo contrario, está bajo licencia de la licencia Creative Commons Reconocimento-NoComercial-CompartirIgual 3.0 Unported. 



\section{Prevenção de recaídas em dependência do tabaco}

Sandra Odebrecht Vargas Nunes Márcia Regina Pizzo de Castro Carlos Roberto de Souza

\section{TRATAMENTOS PSICOSSOCIAIS PARA PREVENÇÃO DE RECAÍDAS EM DEPENDÊNCIA DO TABACO}

Os objetivos das terapias psicossociais de prevenção de recaídas em dependência de nicotina é ensinar formas de evitar a recaída no uso de tabaco em situações de alto risco. Muitos pacientes recaem nos primeiros seis a doze meses após terem tentado parar de fumar. Se o paciente recai, os profissionais da saúde necessitam encorajá-lo a recomeçar. Terapias comportamentais e grupos de apoio podem melhorar as taxas de abandono. (MALLIN, 2002).

Nas terapias cognitivo-comportamentais, os pacientes identificam as situações de alto risco de recaídas e desenvolvem estratégias de manejo. O planejamento de estratégias comportamentais de enfrentamento inclui retirar-se da situação, usar comportamentos substitutos ou desenvolver habilidades (assertividade, habilidade de recusa, manejo de tempo) para gerenciar os fatores que desencadeiam o fumo. $\mathrm{O}$ enfrentamento cognitivo inclui identificar e questionar pensamentos mal-adaptativos e substituí-los por padrões de pensamento mais efetivos (lembrarse de porque é importante parar de fumar ou de que a vontade vai passar), ou tentar impedir que um lapso se torne uma recaída. (AMERICAN PSYCHIATRIC ASSOCIATION, 2008).

Fumantes em manutenção devem ser monitorados quanto aos progressos e dificuldades enfrentados, através de consultas e/ou contatos telefônicos para prevenção da recaída. O paciente precisa se conscientizar de que o tabagismo é uma doença crônica, e que não deve acender um cigarro ou dar uma tragada, pois poderá voltar a fumar. Para evitar recaída, o paciente deve ser estimulado 
a identificar as situações rotineiras que o colocam em risco de fumar e a traçar estratégias para enfrentar essas situações. Se, por acaso, a recaída acontecer, ela deverá ser aceita pelo profissional sem críticas, mantendo a atmosfera de confiança e apoio já demonstrada anteriormente. (REICHERT et al., 2008).

As taxas de abandono após seis meses para terapias comportamentais para tratar indivíduos com dependência à nicotina geralmente variam de $20 \%$ a $25 \%$, ou são cerca de duas vezes mais altas do que as taxas de abandono com indivíduos do "grupo controle”. (AMERICAN PSYCHIATRIC ASSOCIATION, 2008).

\section{PROCESSO DE RECAÍDA: FATORES DESENCADEANTES}

Os pacientes que fumam podem expressar sentimentos ou temores que servem de obstáculos para a cessação do tabagismo. As preocupações mais comuns são o medo de ganhar peso, o medo da abstinência e o de fracassar. A exacerbação dos sintomas psiquiátricos tende a ser um obstáculo adicional para pacientes psiquiátricos. Entretanto, pode-se lidar com a fissura, com o medo de ganho de peso, do fracasso e o medo de agravamento de problemas psiquiátricos com abordagens de resolução de problemas, aumento de monitoramento médico e terapias comportamentais. (AMERICAN PSYCHIATRIC ASSOCIATION, 2008).

As técnicas cognitivo-comportamentais ajudam o fumante a modificar o padrão de conduta no consumo de tabaco, evitando as situações vinculadas às recaídas. Isso se reflete tanto no aprendizado para resistir à compulsão por fumar quanto na adoção de estratégias que se contraponham ao ato de fumar. $\mathrm{O}$ apoio social consiste em reforçar as motivações para parar, fortalecer as vantagens da cessação, aumentar a autoeficácia, combater crenças e racionalizações em torno do consumo, prevenir problemas residuais da cessação (aumento ponderal, irritabilidade, humor negativo) e apoiar o fumante a resolver a ambivalência, se a motivação declinar. (REICHERT et al., 2008). 


\section{AVALIAÇÃO DAS SITUAÇÕES DE ALTO RISCO}

Situação de alto risco significa qualquer determinante interno (psicológico) ou externo (ambiental) que ameace a percepção de controle (autoeficácia) do indivíduo.

As situações de risco de recaída podem ser estados emocionais negativos, conflitos interpessoais e pressão social.

Estados emocionais negativos são aquelas situações nas quais os indivíduos estão vivenciando um estado de humor ou sentimento negativo ou desprazeroso, como frustração, raiva, ansiedade, depressão ou tédio, antes ou no momento do primeiro lapso. Conflitos emocionais são aquelas situações que envolvem um conflito em andamento associado a qualquer relacionamento interpessoal. As pressões sociais são aquelas situações na quais o indivíduo está respondendo à influência de outra pessoa. (BORDIN; FIGLIE; LARANJEIRA, 2004).

Para identificar as situações de alto risco, o que estava fazendo, quais sentimentos envolvidos e desenvolver estratégias de enfrentamento, o paciente deve desenvolver o automonitoramento, anotando cada cigarro fumado, a situação e o sentimento naquele momento. Com esses dados, podemos estabelecer as estratégias junto com o paciente para as situações apresentadas. O cigarro nos serve como uma bengala, como um amortecedor para as emoções sejam elas desagradáveis ou não. O fumante deve, também, avaliar os condicionamentos com o hábito de fumar, como: fumar e tomar um cafezinho; fumar após refeições; fumar antes de dormir, etc. (PRESMAN, 2006).

Para se ter noção do que ocorreu no momento da recaída e ter argumentos convincentes para ajudar o paciente a começar de novo, o profissional de saúde pode fazer as seguintes perguntas (MEIRELLES; GONÇALVES, 2004):

1. O que aconteceu?

2. O que estava fazendo nessa hora?

3. Como se sentiu ao fumar seu primeiro cigarro? 
4. Você já pensou em nova data para parar de fumar?

Comumente, alguns fatores estão ligados à vontade de fumar, tais como após as refeições, café, bebidas alcoólicas, convívio com fumantes, tédio, estados emocionais negativos e/ou positivos, etc. Após identificar as situações de risco, cabe aos dois, profissional de saúde e paciente, determinar a forma mais adequada de enfrentálas.

O ideal seria evitar as situações de risco, mas nem sempre isso é possível. Nas primeiras semanas, deve-se evitar beber café, substituindo-o por suco ou água; da mesma forma, deve-se evitar conviver com fumantes.

Esses pacientes devem ser esclarecidos sobre as circunstâncias que os levaram à recaída sem críticas, e estimulados a tentar outra vez. Uma vez que a recaída faz parte do tratamento, as pesquisas mostram que grande parte dos ex-fumantes tentou pelo menos três vezes antes de conseguir. Deve-se transformar o fracasso em aprendizado, para não se cair nas mesmas armadilhas na próxima tentativa. (MEIRELLES, CAVALCANTE, 2006).

\section{ESTRATÉGIAS DE ENFRENTAMENTO}

Se o indivíduo é capaz de executar uma resposta de enfrentamento cognitiva ou comportamental eficaz diante da situação de alto risco (ser assertivo, por exemplo, no combate à pressão social), a probabilidade de recaída diminui significativamente. Aquele que lida eficazmente com a situação tende a experimentar um senso de domínio ou controle. Essa sensação de domínio cria, no indivíduo, uma expectativa de que será capaz de lidar bem com as próximas ocasiões À medida que a duração da abstinência ou período de uso controlado aumenta, e ele é capaz de lidar efetivamente com mais situações de alto risco, a percepção de controle aumenta de uma forma cumulativa e a probabilidade de recaída diminui proporcionalmente. (BORDIN; FIGLIE; LARANJEIRA, 2004). 
Os profissionais da saúde podem utilizar-se de estratégias para prevenirem as recaídas. Poderão encorajar os pacientes a identificar as pistas e gatilhos e decidir as estratégias de enfrentamento. (OKUYEMI; NOLLEN; AHLUWALIA, 2006).

O sucesso do tratamento pode ser avaliado através da abstinência e/ou ausência de recaídas. Mas isso também se vincula a outros fatores, e o principal deles é a adesão do paciente.

O risco de recaída varia com o tempo do tabaco, da idade de início do consumo, da ansiedade e da depressão, pois tais fatores dificultam a cessação do tabagismo.

Quanto mais cedo se associa alguns comportamentos ao hábito de fumar, mais o fumante passa a agir em função do cigarro. $\mathrm{O}$ fato de se fumar sempre a mesma quantia de cigarros pode estar relacionado à dependência física e psicológica, ou até mesmo ao medo de tentar e não conseguir parar com o vício.

Todo indivíduo precisa se preparar para enfrentar processos de abstinência e cessação do cigarro uma vez que, antes da parada total, todo fumante faz tentativas no sentido de melhorar e superar a recaída.

Muitas pessoas que buscam tratamento não se acham capazes de chegar até o fim, devido a insucessos anteriores. $\mathrm{O}$ ambiente também é um fator a ser considerado, pois pode interferir no esforço do fumante devido a pessoas, participantes desse mesmo, ambiente terem o hábito de fumar.

Técnicas de controle comportamental são utilizadas no sentido de auxiliar o fumante, tirando de perto dele tudo o que possa lembrar tanto o cigarro, como o hábito de fumar, até mesmo o local que associe ao cigarro.

O quadro 18 sumariza uma análise funcional de situações de risco de recaídas e estratégias de enfrentamento. 


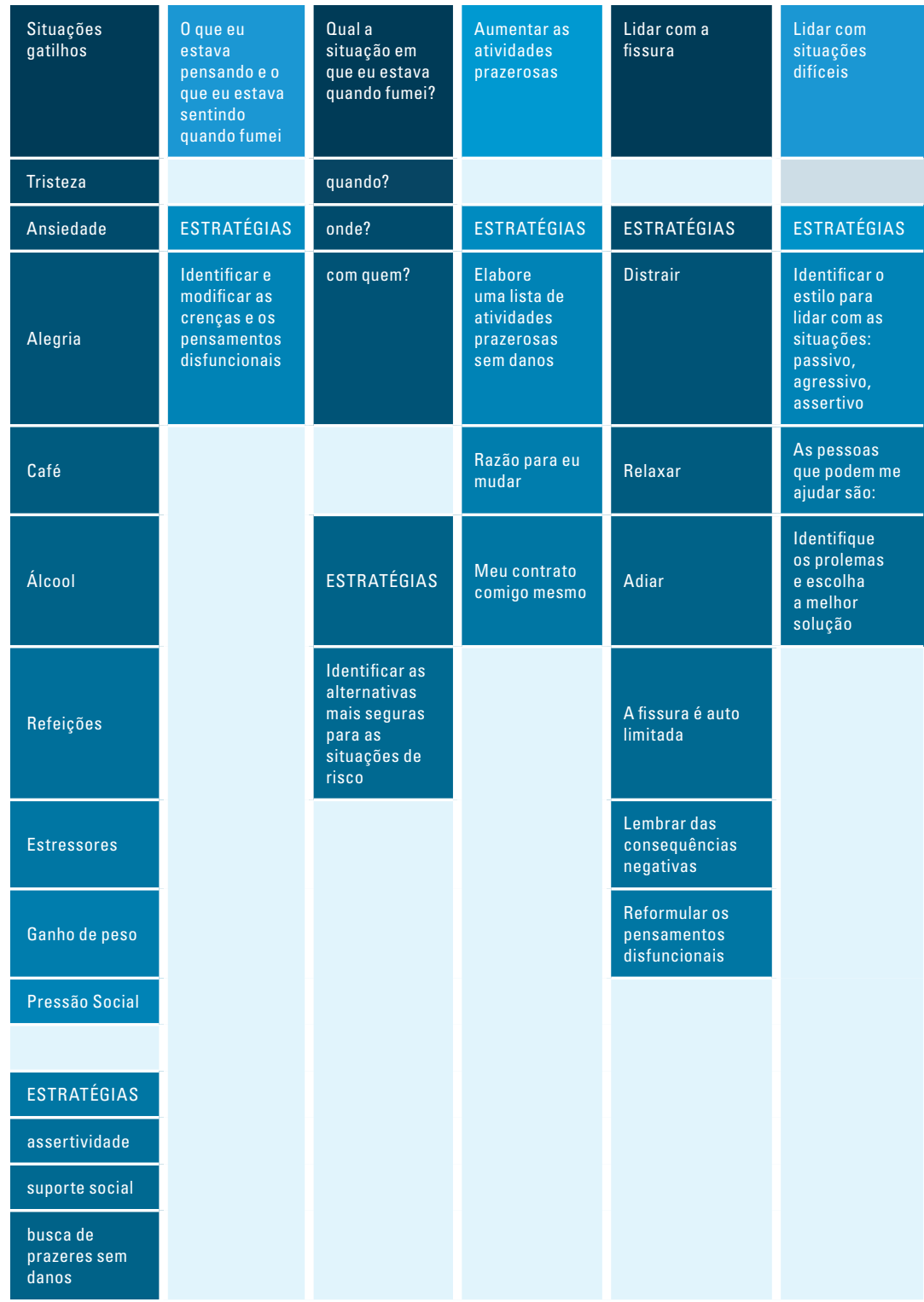

Quadro 18 - Situações de risco de recaídas e estratégias de enfrentamento. 
Para que o fumante se torne mais forte e enfrente situações, é possível aconselhá-lo e também ajudá-lo a lidar com a vontade de fumar. Aspectos sociais e psicossociais indevidos podem levar a uma elevação da ansiedade e estresse e, se não forem bem trabalhados, poderão levar o indivíduo à recaída.

Várias estratégias de enfrentamento têm produzido bons resultados no tratamento e em sua manutenção. Dentre elas, as mais funcionais e objetivas são: o trabalho com o sentimento e as emoções através da verbalização; a assertividade; a prática de exercícios físicos; a mudança de hábitos e costumes; a dieta eficaz; o alerta quanto aos malefícios provocados a fumantes passivos; a participação nos grupos de autoajuda e o incremento da motivação no processo de superação da dependência.

A recaída está intimamente ligada e constatada de forma geral nos três primeiros meses de tratamento. Necessária se faz a valorização do enfrentamento e a compreensão do estado, que é previsto no processo de tratamento, não sendo algo para a catastrofização, porém algo natural, uma escala a mais das etapas a serem superadas.

A seguir, um breve apanhado de como auxiliar o paciente a parar de fumar, por (GLYNN; MANLEY apud SOLHA, 2000, p. 41):

- $\quad$ arguir se o paciente deseja parar de fumar em todas as consultas; se não desejar, fornecer folhetos com informações gerais sobre o fumo; se deseja, colocar-se à disposição para auxiliá-lo;

- $\quad$ aconselhar todos os fumantes a parar de fumar;

- ajudar o paciente a parar com informações precisas, prescrição de medicamentos e materiais de autoajuda;

- acompanhar, marcando consultas futuras; associar a farmacoterapia com a psicoterapia disponível.

Segundo Solha (2000, p.42): 
Um dos maiores efeitos deste tipo de aconselhamento é motivar os pacientes a pararem, se estes não conseguirem sozinhos, poderão ser encaminhados a serviços especializados. Uma das desvantagens é o desinteresse de muitos profissionais por este assunto, desconfiança nas habilidades do paciente e baixa remuneração (o que para alguns, justifica um atendimento extremamente rápido).

O cronograma abaixo, descrito por Laranjeira (apud SOLHA, 2000, p.42), pode ser usado por profissionais da saúde para avaliar a motivação do paciente e para a realização dessa intervenção:

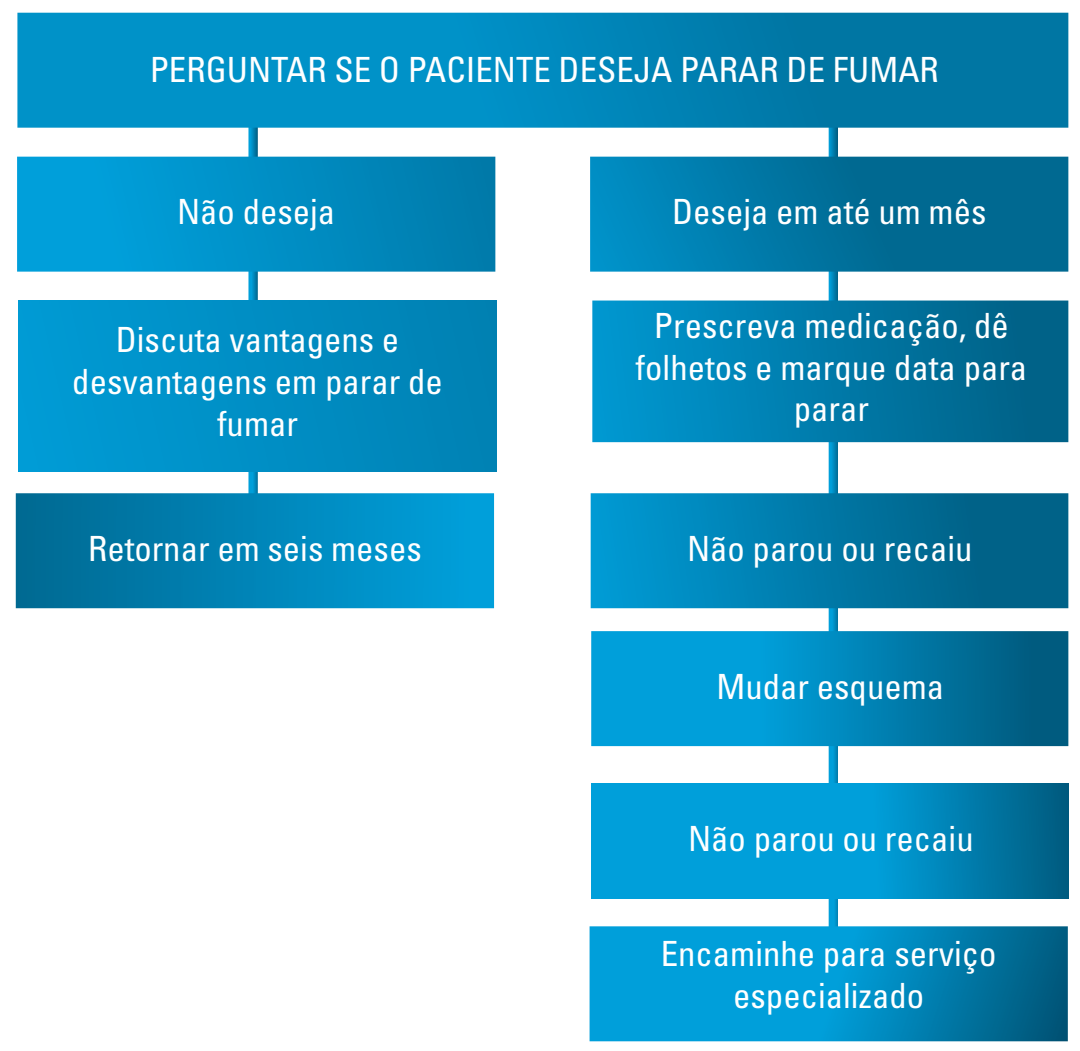

Figura 3 - Motivação do paciente. 


\section{PREVENÇÃO DE RECAÍDA}

A preparação para a terminação de um processo terapêutico que tem uma elevada eficácia na abordagem dentro dos princípios da terapia cognitivo-comportamental formulada por Alan Marlatt nos idos dos anos 1980, com enfoque na reestruturação cognitiva e mudanças comportamentais, com programação de acompanhamento e reforço nas sessões, tem como prioridade o acrescer consciente e a decisão do paciente com relação à sua problemática, sem fixação na abstinência, preocupado com a redução de danos. $\mathrm{O}$ tratamento nada mais é do que um sistema organizado voltado para sustentar a alteração para melhor dos comportamentos de danos, buscando evitar condutas de risco, a sustentação maior no treino de habilidades e na consciência da situação pelo paciente. Possui fases de reconhecimento, de evitação e de enfrentamento.

A utilização de métodos de avaliação para situações consideradas de alto risco, tais como: percepção fixa do paciente, mensuração da alta eficácia, história de vida, experiências descritas das recaídas e autoaconselhamento são sumarizadas na figura 4. Tudo isso dentro do aspecto interno (psicológico) ou externo (ambiental), conforme o modelo cógnito-comportamental do processo de recaída: 


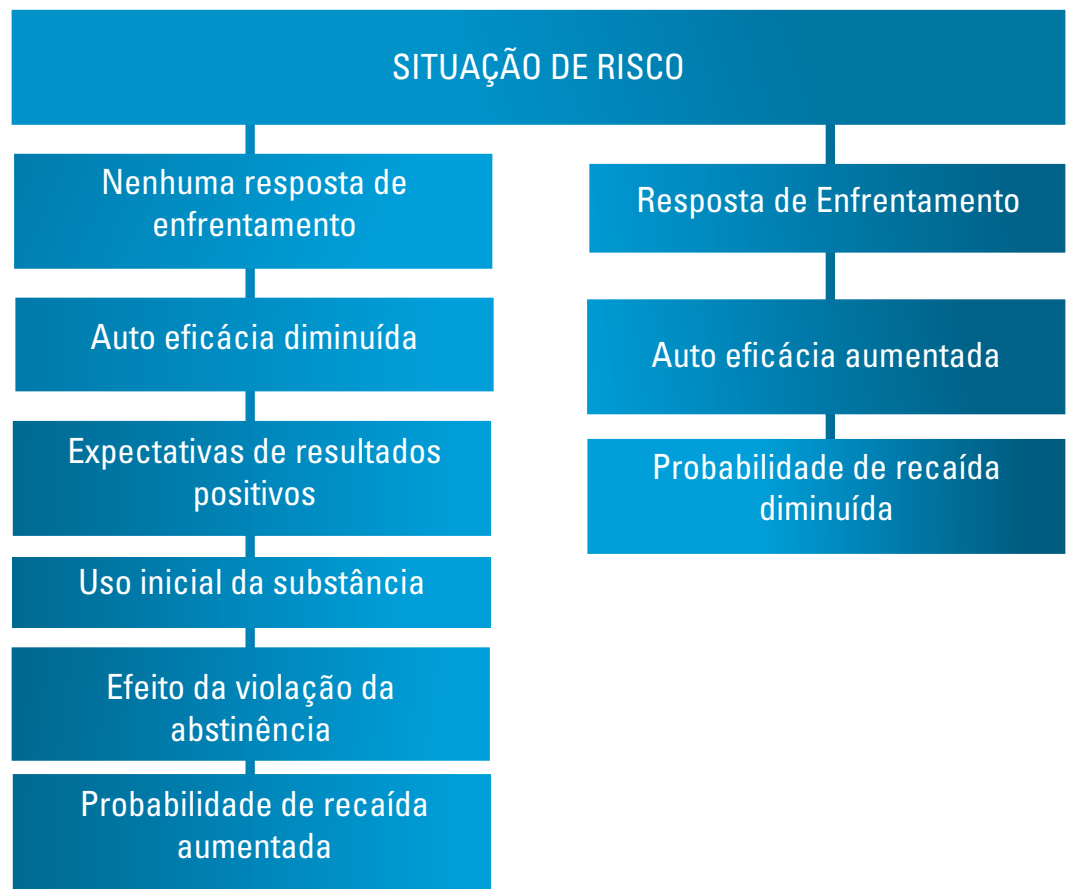

Figura 4- Avaliação das situações de risco de recaída e resposta de enfrentamento.

Fonte: Marlatt (apud BORDIN; FIGLIE; LARANJEIRAS, 2004, p. 252).

A prevenção de recaída apresenta alguns sintomas, assim elencados:

- mecanismo de negação do estresse, comum no processo mutacional;

- dever irreversível com o estado de abstinência;

- $\quad$ irritabilidade e conflitos com os envolvidos no tratamento;

- $\quad$ falta de sentido com relação à vida;

- $\quad$ esquiva, ódio, raiva, medo e ilusão;

- não admissão de apoio (introspectivo e/ou violento); 
- $\quad$ sentimento de que "sem o tabaco fica pior";

- autopiedade neurótica consistente;

- enfraquecimento da autoconfiança;

- $\quad$ conflito sem razão: do mundo, de si e descrença com relação ao futuro;

- não adesão e desistência do tratamento;

- $\quad$ atos de competição e;

- $\quad$ imponência

Esses sintomas necessitam ser revisados e praticados constantemente, em suas estratégias e técnicas, até porque, para o nosso paciente, não interessa qual a abordagem utilizada, e sim a eficácia/sucesso do tratamento e, de preferência, que haja o menor sofrimento ou amargura, dentro de um aspecto empático extremamente respeitoso, em que os compromissos são divididos (50\% em média) entre paciente e terapeuta, com a responsabilidade também dividida na busca da eficácia.

Os desafios dos pensamentos automáticos negativos têm de estar afinados com a consequente terminação e ainda com o monitoramento preestabelecido dos prazos demarcados ou datas oportunas.

Concluindo, não há uma programação. As técnicas de enfrentamento na prevenção de recaída são aplicadas conforme as diferenças individuais de cada ser, em conjunto com a experiência e habilidade do profissional de treinamento/apoio. O estilo de vida renovado faz a diferença no processo.

\section{REFERÊNCIAS}

AMERICAN PSYCHIATRIC ASSOCIATION. Diretrizes par o tratamento de transtornos Psiquiátricos compêndio 2006. Porto Alegre: Artmed, 2008. 
BORDIN, S.; FIGLIE, N.; LARANJEIRA,R. Prevenção de Recaídas. In; FIGLIE, N.; BORDIN, S.; LARANJEIRA, R. Aconselhamento em dependência química. São Paulo: Roca, 2004.

FIGLIE, Neliana Buzi; BORDIN, Selma; LARANJEIRA, Ronaldo. Aconselhamento em Dependência Química. São Paulo: Roca, 2004.

GLYNN, T. J.; MANLEY, M. W. How to help your patients stop smoking: a National Cancer Institute manual for physicians. U.S. Departament of Health and Human Services. ANtional Institute of Health, NIH publications, p.89-3064, 1998.

MALLIN, R. Smoking cessation: Integration of behavioral and drug therapies. American Family Physician. v.65, p.1107-14, 2002.

MARLATT, G. A. Prevenção de Recaída: racionalidade teórica e visão geral do modelo In: MARLATT, G. A.;GORDON, J. R. Prevenção de RecaídaEstratégias de manutenção no tratamento de comportamentos adictivos. Porto Alegre: Artes Médicas Sul, 1993.

MEIRELlES, R.; CAVALCANTE, T. Quais políticas de controle do tabagismo um país deve ter para chegar a um tratamento eficaz? A perspectiva governamental. In: GIGLIOTTI, A.; PRESMAN, S. (Org.). Atualização no tratamento do tabagismo. Rio de Janeiro: ABP-Saúde, 2006. p.171-189.

; GONÇALVES,C. M. Abordagem cognitivo-comportamental do fumante. J. bras. pneumol., [s.1.], v.30, p.50-59, suppl.2, 2004.

OKUYEMI, K.; NOLLEN, N.; AHLUWALIA, J. Interventions to facilitate Smoking Cessation. American Academy of Family Physicians,v.74, p.26271, 2006.

PRESMAN, S. Intervenção intensiva e terapia de grupo. In; GIGLIOTTI, A.; PRESMAN, S. Atualização no tratamento do Tabagismo. Rio de Janeiro: ABPSaúde, 2006.

REICHERT, J. et al. Diretrizes da SBPT - Diretrizes para cessação do tabagismo - 2008. J Bras Pneumol, v.34, n.10, p. 845-880, 2008.

SOLHA, Raphaela K. de T. Desintoxicação em Fumo. In: LUIS, Margarita Antonia Villar; SANTOS, Manoel Antonio dos (orgs.). Uso e Abuso de Álcool e Drogas. Ribeirão Preto, SP: FIERP-EERP - USP / FAPESP, 2000. 